\title{
Measurement of subresolution terrain displacements using SPOT panchromatic imagery
}

\author{
Satellite-derived imagery can be used to meas- \\ ure subresolution horizontal terrain displacements \\ that are associated with present-day earthquakes, \\ sand dune migration, coastal processes, glacial mo- \\ tion, and, perhaps, preeruptive volcanic processes. \\ This use of these data will increasingly facilitate the \\ understanding of natural hazards and the determi- \\ nation of the rates of many environmental processes \\ worldwide.
}

\section{Introduction}

Satellite image data provide maps of the surface of the Earth that are detailed, uniform, and spatially comprehensive over wide areas. These attributes allow researchers to use these data in order to solve significant problems in the earth sciences that are not practical to solve by any other means. Research is now underway to extend the use of satellite imagery to the detection and measurement of surface processes occurring at scales below the limits of resolution.

The premise of the method is that areally extensive. subresolution spatial differences in ground patterns between images acquired at differing times can be measured accurately to high precision and also can be distinguished from systematic image differences, such as those due to sensing-system attitude variations. The idea for this approach was conceived while considering the tectonic effects of the October 17, 1989, Loma Prieta ("San Francisco") earthquake and their possible effects upon image data (Crippen. 1990). Thus far. testing of a preliminary algorithm shows that the approach is reasonable.

The data currently used are from the French SPOT satellite, which has a "pushbroom" sensor array that provides $10-\mathrm{m}$ panchromatic imagery. In some cases, the terrain displacements one may seek to measure may be a small fraction of the pixel size. In other cases, the displacements may exceed the pixel size, but one may seek subpixel precision. The general approach is to match spatially the "after" image to the "before" image at each point on a grid by iteratively interpolating one and testing its correlation with the other. The statistical sample of each image at each grid point is typically thousands of pixels. Spatial variations in the results correspond both to changes on the ground ("geometric signal") and to image distortions ("geometric noise"). However, most image distortions are attributable to sensor-attitude variations that can be identified and removed through recognition and measurement of their distinct spatial characteristics. This allows us the possibility of isolating and revealing patterns of environmental change.

Note that the objective is not to resolve subresolution objects, nor is it necessary to resolve subresolution objects. Instead, the goal is to detect and measure surface processes by matching statistically the radiometric patterns in the data that differ spatially in a consistent direction over many pixels.

\section{Advantages of imageodesy}

The name coined for this method is "imageodesy." which is both a concatenation of "image geodesy" and a partial acronym for "Image Multitemporal Analysis Geodesy." Clearly, there are limitations to the application of imageodesy, but it also has several potential advantages over currently available alternatives.

In the study of seismotectonic deformation along a fault, for example. comparable methods require the establishment of benchmarks at selected locations followed by labor-intensive surveys. In order to map the strain field associated with an earthquake, scientists must wait years, decades, or centuries for an earthquake to occur on that segment of that fault. In contrast, by using satellite image data, an earth scientist need not anticipate the time and location of an earthquake nor concentrate his effort in any one region in the hope of an opportunity to collect data (that is, an earthquake) during his lifetime. The before images are available and continue to accumulate in image libraries (collected by other people for other purposes at no cost to the imageodesist until needed), and the after images can be ordered as needed for any location in the world. Problems of accessibility due to terrain or political factors do not exist, and the data are spatially comprehensive.

Similar advantages of labor savings, spatial uniformity and detail, and global coverage and access are applicable to studies of sand dunes, coastal processes, glaciers, and volcanic processes. Significantly, imageodesy enhances the value of imagery collections as historical records of environmental change.

\section{General approach}

\section{Fundamental concept}

Basically, the imageodesy method relies upon the fundamental statistical concept of a normal distribution. The essence of this concept is that accurate and precise measurements can be obtained statistically from a set of measurements that are individually unreliable and even relatively crude. If measurements have errors that are random, then their probability distribution is symmetrical and bell-shaped and has a peak at the true value. If enough measurements are made, then the shape of the "bell" can be defined sufficiently well to determine the peak accurately. Thus, the probability of accuracy of the statistical determination (at any given level of precision) improves as the number of measurements increases.

Each pixel value is a measure of radiance weighted at subresolution scales by the point-spread function that is imposed by the atmosphere and sensor optics. Each pixel value is, therefore, variable in relation to its geographic position at subresolution scales and thereby indirectly measures geographic position. Imageodesy typically compares thousands of pixels in the before image to thousands of pixels in the after image in determining each displacement vector, 
$d$ it can, as a result, be capable of high accuracy at high levels of atial precision.

Clearly, the problem faced is not this simple. The measurement , ors that must be overcome (radiometric and geometric noise in the lage) are not fully random, and other data inadequacies are possi¿. These difficulties are discussed in the "Sources of error" section.

\section{fasic algorithm}

he idea for the procedure is an outgrowth of image-matching work lat was developed and used to register misaligned bands in a multijectral data set (Crippen. 1987). A vector is calculated at each node $n$ a grid by the use of (for example) 1 square kilometer of data 10,000 pixels) from each image. Several improvements can be rpected as this research continues, but the basic correlation "hill limbing" routine for each vector, as used in initial tests, is as ollows. (1) The before and after scenes are best-fit matched to the earest pixel, as determined by visual inspection. (2) The before mage is used still as the mapping base and is held "constant." (3) For ach vector determination, $100 \times 100$ pixels of the before image are ompared statistically for maximum correlation to a moving template if $100 \times 100$ pixels in the after image, as follows.

For full-pixel steps:

A) Correlation is determined at the starting (full-scene visual) match position and at the 24 neighboring full-pixel steps. which results in a $5 \times 5$ array of correlation coefficients.

B) If the maximum correlation in the array is not at the starting match position, a vector is "drawn" (in effect) to the point of maximum correlation, and a new $5 \times 5$ array of correlation coefficients is calculated (without redundancy) at that point.

(C) Maximum correlation is determined again, and the vector is "redrawn," if necessary. (Further iterations are allowed but were not necessary in the initial tests. As verified visually, the best-fit starting position provided image registration to within a few pixels at all locations within the scene.)

For subpixel steps:

(A) Repeated bilinear interpolation of the after image is applied in order to create a set of images that are translationally shifted by half-pixel steps for comparison to the before image. These include the peak-correlation full-pixel shift location and its eight neighbors (a $3 \times 3$ array of correlation coefficients). The highest correlation in this array is determined, and the vector is "redrawn" to it, if necessary.

(B) Quarter-pixel image shifts that include the peak-correlation halfpixel shift location and its eight neighbors (again, a $3 \times 3$ array) are calculated, their peak correlation value is determined, and the vector is again "redrawn," if necessary.

(C) This routine is repeated in seven steps down to shift steps of $1 / 128$ pixel (about $8 \mathrm{~cm}$ on the ground). Not surprisingly, 1/128 pixel appears to be well beyond the random noise level of precision, as shown by separate tests (see "Indication of precision limit" section).

After the full array of vectors across the image is generated, trends that are clearly attributable to differences in data collection between the before and after images are characterized and removed. If these trends are related to detector array distortions, then they are distinctly constant along the satellite path (and appear as differences among entire columns of pixels). If they are related to attitude variations through time, then they are distinctly constant across the satellite path (and appear as differences among entire rows of pixels). If they are related to static differences between scenes, such as a difference in scale, then they form distinctive two-dimensional patterns across the entire image. Thus, these trends are spatially distinct and generally can be removed in order to isolate and reveal image differences that are due solely to ground deformations.

\section{Results of initial tests}

A test site at the Superstition Hills, an arid area in southernmost California, USA. was chosen in order to attempt the detection of fault offsets related to the magnitude 6.6 earthquake on November 24,1987 . The maximum combined coseismic and postseismic offsets along the Superstition Hills fault for this quake are on the order of 1 m (McGill and others, 1989; Burgmann and others, 1989), which posed a challenge for the imageodetic method at its current level of implementation. The available before image was acquired by the SPOT-1 satellite on November 11, 1987, and the after image selected was acquired on February 21, 1989. Both scenes were acquired from nearly the same orbital position and so provided matching look angles. Although the dates were not optimal for matching the sun positions and other seasonal effects, the image dates were approximately symmetrical around the winter solstice such that the sun positions were similar. Also, the arid and rugged, yet fairly low relief. character of this terrain provided strong feature patterns that allowed us to match features statistically, and yet they minimized the impact of potential differences between the scenes.

Unfortunately, the fault offsets were not clearly distinguishable in the imageodetic results. This is likely due to the fairly simplistic implementation used thus far and to the small size of the offsets, which are only slightly larger than the best precision expected from the technique under optimal conditions. Several refinements to the technique, now under investigation, may prove critical to this particular tectonic application.

Fortunately, however, the imageodetic method was demonstrated successfully in this same image pair by the subresolution measurement of barchan and longitudinal sand dune migration in an area immediately west of the Superstition Hills. The dune movement was detected because the dunes and their shadows (in contrast with the underlying terrain) form the dominant radiometric patterns in this local area. Figure 1 shows the SPOT panchromatic imagery for the dune site (top) and displays the corresponding temporal difference vector pattern (bottom). Note that the large, anomalous vectors correspond distinctly to the geographic positions of the dunes, point downwind for the barchan dunes (as verified by dune morphology), and measure up to $7 \mathrm{~m}$. This amount of movement is very reasonable for the timespan between the images ( 15 months) for dunes of this size (about $50-70 \mathrm{~m}$ across). Movements of one nearby dune (in an area likely to have stronger winds) have averaged $15 \mathrm{~m}$ per year over past decades (Shelton, 1966).

\section{Error types and limitations}

\section{Sources of error}

The primary challenge in this work is to overcome measurement errors of a wide variety such that adequate levels of precision and accuracy can be attained in the image comparisons. Sources of error are of two primary types: radiometric and geometric problems.

Errors due to radiometric problems result primarily from differences across the detector array, difficulty in detecting patterns in the image (signal-to-noise inadequacies), and radiometric changes in scene content between the dates of image acquisition. SPOT image data have very high radiometric quality; however, radiometric quality requirements for imageodesy are unusually high. 

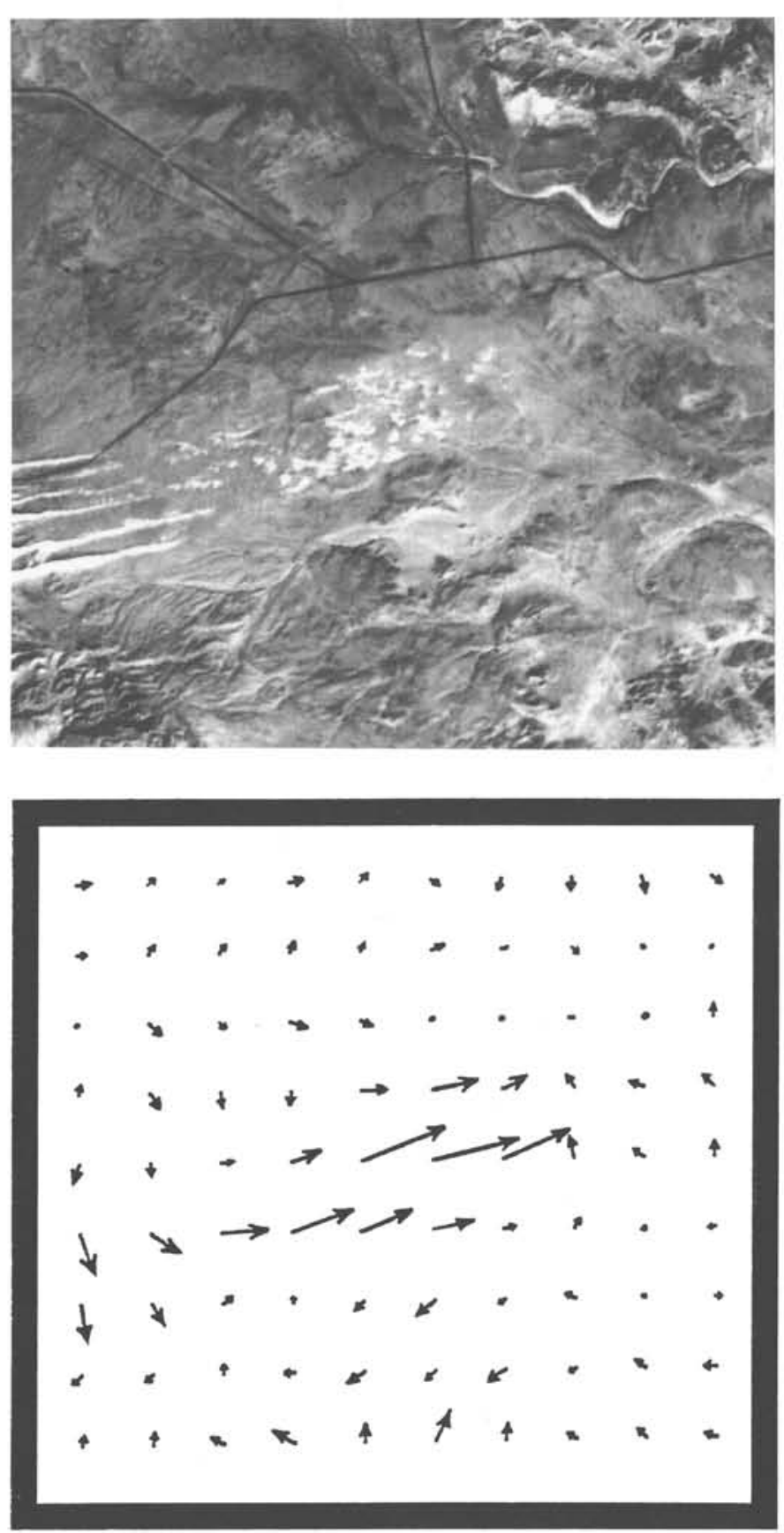

Figure 1.-Top: SPOT panchromatic image of Superstition Hills sand dune site in southernmost California, USA (average of before and after scenes). (C) CNES. Bottom: Vector ray of imageodesy results of dune site. Array spacing is $500 \mathrm{~m}$, and vector exaggeration factor is 100 . Note that anomalous vectors closely correspond to the dunes and indicate up to about $7 \mathrm{~m}$ of displacement. Background noise is mostly less than $1 \mathrm{~m}$.

Minor differences across the detector array (which may be significant in some cases) are evident as vertical striping, especially in data that have been filtered to enhance high spatial frequencies. A time-variable error that results in horizontal striping is also evident. It may be possible to adjust for both of these error types by character- izing and correcting them on a column-by-column and row-by-row basis. In order to allow this and to avoid unnecessary image degradation due to resampling, imageodesy uses unrectified "level $1 \mathrm{~A}$ " SPOT data.

An inherent limitation of the method is that patterns in the image data are required in order to conduct image comparisons. In some cases, the terrain will be flat and featureless and very difficult to match between images (signal variance will be inadequate). A map of correlation coefficients can be used to identify such problem areas, but it is unlikely that this problem can be fully solved. Fortunately, such areas are seldom of interest anyway.

Radiometric differences between the scenes can be a problem also. Natural vegetation can grow or die, drainage channels across alluvial terrains can migrate, and roads can be modified. In some cases, entirely new features will not be problems because they will not spatially match anything in the older scene and will not, therefore, spatially bias the correlation patterns (the peak correlation will decrease in magnitude but will not shift spatially). In general, however, major radiometric features should be identified by image differencing and then removed from the correlation procedures by image masking. In areas where radiometric change is pervasive (as, for example, agricultural fields), the subresolution matching of edges in the image (such as field boundaries) may be possible as an alternative procedure.

The problems of "geometric noise" also are critical but not insurmountable. Sun-angle and view-angle differences between scenes should be avoided. Shadows will not match between scenes having differing sun positions. Likewise, radial distortion of topographic features in images will differ between images of differing view angles. In many cases, one must, therefore, compare scenes taken from the same orbit path (SPOT is a pointable sensor) on approximately the same day of the year. SPOT is in a sunsynchronous orbit, so matches of time of day are not a problem.

The other major source of geometric noise results from sensorattitude (and altitude) differences during the two periods of image acquisition. Variations between scenes in yaw, pitch, and roll (and scale) are all possible. Ancillary data on these variations are provided on SPOT digital data tapes and are described in the "SPOT User's Handbook" (CNES, 1988). However, the best way of dealing with these problems is to identify, measure, and remove their fairly distinct spatial patterns in the initial imageodetic results, as discussed in the "Basic algorithm" section. In initial tests, the primary differences were found to be constant (static) differences in yaw and scale and constantly changing (dynamic) differences in pitch and roll.

\section{Indications of precision limit}

The precision of image matching varies as a function of the image signal-to-noise ratio, the number of pixels involved, the image pattern textures, and the interpolation method (Forstner, 1982; Sutton and others, 1988). However, a general statement on precision may be possible.

Tests were used to map out broad arrays of correlation coefficients at each level of precision for a few sites. The resulting maps showed correlation increasing smoothly to a peak at the coarser levels of precision, but it increased irregularly at the finer levels of precision, and a transition took place at about $1 / 16$ to $1 / 32$ of a pixel. This may indicate the limit of precision for an accurate single-vector result, exclusive of the problems of broader image distortions and exclusive of image preprocessing steps or image-matching algorithm changes that possibly could improve these results.

Also encouraging is the work of other researchers who have used image matching and analyses in different contexts. Although they have used a variety of data types and processing routines, 


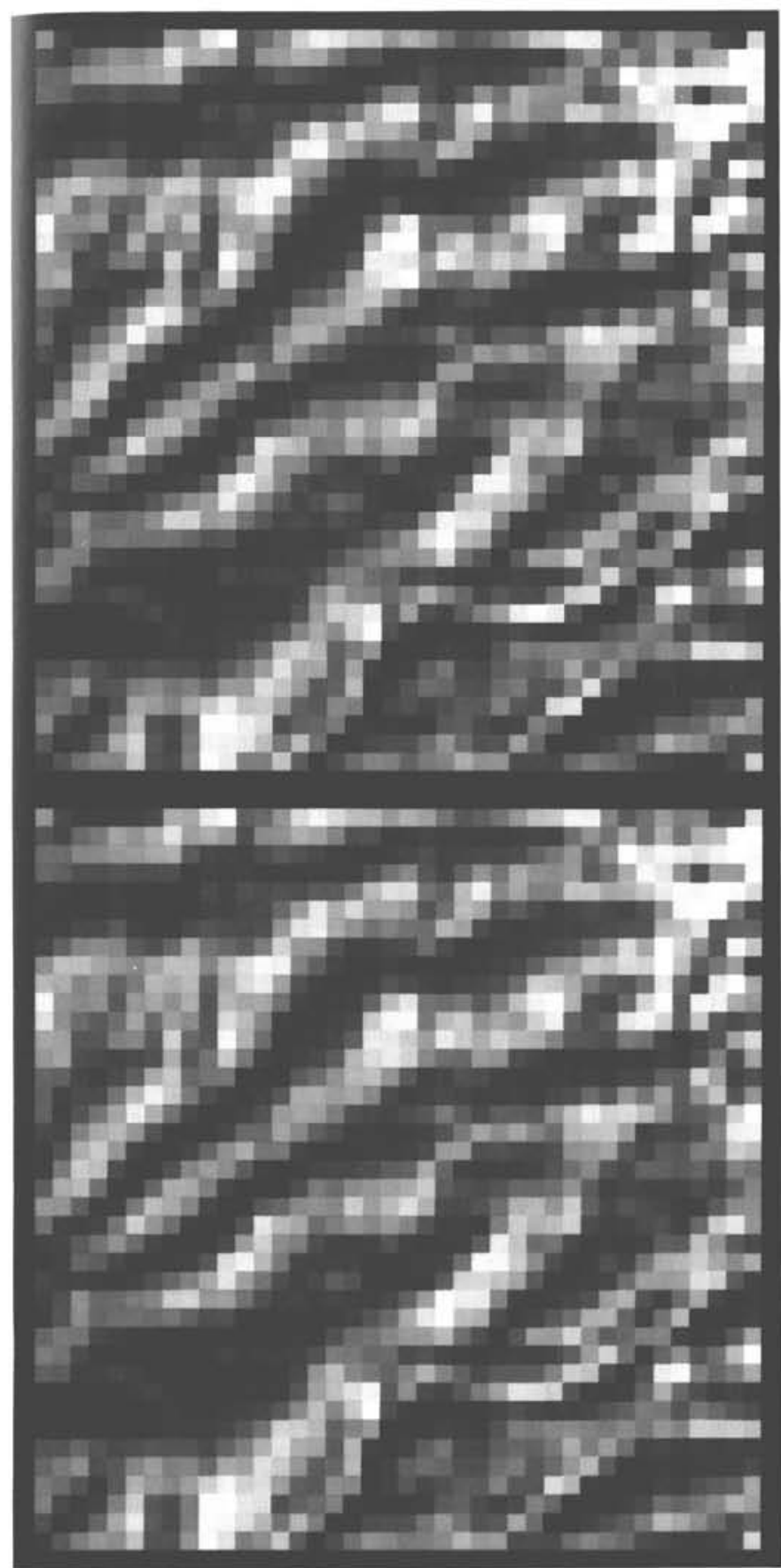

Figure 2.-Simulations of left-lateral (top) and right-lateral (bottom) subresolution fault offsets showing before images in cyan and after images in red. In each case, the fault is located at a color fringe boundary, and the sense of offset is indicated by the locations of color fringes relative to shadows (see text).

several have found a limit of precision at about 1/20 of a pixel (Qi and Huhns, 1986; Berenstein and others, 1987; Mort and Srinath, 1988; Morgan and others, 1989; Havelock, 1989; Lyvers and others, 1989; $\mathrm{Li}, 1990$ ). A precision of $1 / 20$ of a pixel corresponds to $50 \mathrm{~cm}$ on the ground. This precision would be extremely useful for many purposes.

\section{Related image-analysis methods}

In addition to vector calculations, at least two alternative methods are possible for determining relative displacements in multitemporal image pairs. Both involve coregistering the images as closely as possible (by subresolution resampling) in order to provide an overall best fit. One method is to flicker (temporally alternate) the two images (both in gray tones) on an image-display monitor at substantial enlargement so that individual pixels can be seen. The directions and magnitudes of subresolution shifts can be seen easily (barring major radiometric noise), although their significance may be difficult to ascertain in the context of image distortions resulting from attitude variations. The other method is simply to display the two images in complementary colors simultaneously and to judge the patterns of subresolution misregistrations as indicated by color fringes.

Two examples of the color fringe method are simulated in figure 2. A single SPOT image was used (thus avoiding radiometric and geometric noise) and was resampled in order to simulate a fault offset in the after image. The fault runs vertically as a north-south trace (north to the top) directly through the middle of the image, and displacements are uniform within the west block and within the east block. The before image is displayed in cyan (blue and green), and the after image is displayed in red.

In the upper simulation, movement along the fault is left lateral at one-half pixel $(5 \mathrm{~m})$, and the east block has been given perfect registration. Note that the color fringes in the west block demonstrate the general direction of offset (for example, bright red has moved relatively southward and impinged upon dark cyan, which has produced a red color fringe on the north side of each shadow). Also note that the location of the fault is evident as the eastern boundary of the color fringes. Thus, both the location of the fault and its sense of motion can be determined from the image pair, despite the subresolution amount of fault displacement.

In the lower simulation, movement along the fault is right lateral at three-fourths pixel $(7.5 \mathrm{~m})$, the west block again having a misregistration of one-half pixel $(5 \mathrm{~m})$ and the east block now having a misregistration of one-fourth pixel $(2.5 \mathrm{~m})$ in the opposite direction. Note that (1) the color fringes on the west block have reversed in color compared to the upper version (red fringes are now south of shadows), (2) on the same sides of shadows, the color fringes are opposites between the east and west blocks (cyan on the north side of shadows on the west block and red on the north side of shadows on the east block), and (3) the saturation of the color fringes is greater on the west block because of its greater offset (misregistration) amount. In this case, the fault can be located by the abrupt changes in the hue of given fringes as the fault is crossed, and the sense of motion can again be determined by the relative positions and hues of the fringes.

These techniques provide useful tools that augment the imageodesy vector method. The vectors provide statistically significant results that can be modeled to remove geometric noise and thereby obtain precise displacement magnitudes and directions over wide areas. Fault breaks in remote locations might be located by the vectors although only to within the $1-\mathrm{km}$ breadth of the imagesampling area. Image-display methods can be used then to locate a fault to within one pixel (10 $\mathrm{m}$ for SPOT images).

\section{Related studies and possibilities}

Image analyses similar to imageodesy have been used previously in the study of cloud motions (Leese and others, 1971), sea-ice movements (Ninnis and others, 1986), sea-surface velocities (Emery and 
others, 1986; Garcia and Robinson, 1989), and glacial ice streams (Bindschadler and Scambos, 1991). The purpose of the current work has been to develop this approach to a level that is useful for solving geologic problems, especially tectonic problems.

The particular challenge of tectonic problems is the small scale of deformation associated with even the largest tectonic events over short time intervals. Maximum fault offsets are typically no more than $8 \mathrm{~m}$ for even the greatest earthquakes. Thus, if we use $10-\mathrm{m}$ resolution imagery, subresolution measurements are essential, which is not the case in the study of clouds, sea surfaces, or glaciers. Further, the lessons learned from the challenges faced in tectonic applications should prove to be valuable across the full spectrum of applications. The importance of the work lies in the understanding of natural hazards, such as earthquakes, and in the determination of the rates of environmental processes in general, particularly as concern grows about global change.

The range of possible applications of imageodesy varies according to sensor characteristics, particularly spectral sensitivities, spatial resolutions, and repeat coverage cycles. One interesting possibility is the acquisition of image pairs that are separated by only a few seconds and are used for the study of rapidly moving features. The SPOT satellite currently provides this capability. It has two image sensors per satellite, which can be used simultaneously. In acquiring panchromatic $(10-\mathrm{m})$ data, the sensors point slightly forward along the satellite path, and in acquiring multispectral $(20-\mathrm{m})$ data. they point slightly backward (CNES, 1988). This results in simultaneous data acquisitions that are separated by about $15 \mathrm{~km}$ (one-quarter scene length) on the ground. At a scene acquisition period of 9.024 seconds, the time difference for the two modes to view the exact same terrain is about 2.3 seconds. One study already has demonstrated that velocities of boats, trains, aircraft. roadway vehicles, and clouds can be determined by using such image pairs (Stern. 1987). Comparable applications having appropriate sensors might allow, for example, nearly instantaneous, precise determinations of sediment or thermal pattern flow in rapidly moving coastal currents.

Another useful application (over a period of days or weeks) could be the detection of topographic changes associated with magmatic migration prior to volcanic eruptions, such as those that occurred at Mount St. Helens in the USA in 1980. Even more applications may become evident as refinements increase the robustness of the technique.

\section{Future work}

Future work on the imageodesy technique will include refinements aimed at assuring high precision and optimum accounting for systematic spatial noise. The former may require filtering of the data, selective masking of temporal differences in land cover, and (or) use of alternative interpolation and image-matching schemes. The latter will require refined models of the possible results of attitude variations and other factors. Much experimentation will be needed.

A particularly alluring goal of this research is to produce a spatially comprehensive map of horizontal terrain displacements associated with at least one large earthquake. So far, only one primecandidate earthquake has occurred since the launch of the first SPOT satellite in 1986, namely, the Luzon, Philippines, earthquake (magnitude 7.9) of July 16. 1990 (Newhall and others, 1990). One useful before image exists, but a cloud-free after image of comparable season and look angle has not become available for this tropical location. Meanwhile, refinements are being developed on the Superstition Hills imagery, the worldwide collection of before images continues to grow, and the next great earthquake gets closer, day by day.

\section{Acknowledgments}

This work was carried out at the Jet Propulsion Laboratory (JPL), California Institute of Technology, Pasadena, California, USA, under a contract with the National Aeronautics and Space Administration. Support for this project was provided by a grant from the JPL Director's Discretionary Fund. Assistance was provided by the JPL Supercomputer User Support Staff. Thanks to Ronald Blom and several other colleagues for their critiques, encouragement, and support of this research.

\section{References}

Berenstein. C.A., Kanal, L.N., Lavine, D., and Olson, E.C., 1987. A geometric approach to subpixel registration accuracy: Computer Vision, Graphics, and Image Processing, v. 40, no. 3, p. 334-360.

Bindschadler, R.A., and Scambos, T.A., 1991, Satellite-image-derived velocity field of an antarctic ice stream: Science, v. 252, no. 5003, p. 242-252.

Burgmann, R., Behr, G., and Bilham, R., 1989, Creep events and interevent slip on the Superstition Hills fault, California: EOS (American Geophysjeal Union Transactions), v. 70, no. 43, p. 1348.

CNES (Centre National d'Etudes Spatiales), 1988. SPOT user's handbook: Reston. Virginia, USA, SPOT Image Corporation. v. 1 and 2.

Crippen, R.E.. 1987, The regression intersection method of adjusting image data for band rationing: International Journal of Remote Sensing, v. 8, no. 2 , p. $137-155$.

- 1990. Concept for the measurement of horizontal terrain displacements associated with the Loma Prieta earthquake of 17 October 1989 using SPOT panchromatic imagery: Geological Society of America Abstracts with Programs, v. 22, no. 3, p. 16.

Emery, W.J., Thomas, A.C., Collins, M.J., Crawford, W.R., and Mackas, D.L.. 1986, An objective method for computing advective surface velocities from sequential infrared satellite images: Journal of Geophysical Research, v. 91, no. C11, p. 12865-12878.

Forstner. W., 1982, On the geometric precision of digital correlation, in Hakkarainen, J., Kilpelä, E., and Savolainen, A., eds., Mathematical models, accuracy aspects and quality control, proceedings of the symposium: International Archives of Photogrammetry, v. 24-III, p. 176-189.

Garcia. C.A.E., and Robinson, I.S., 1989, Sea surface velocities in shallow seas extracted from sequential Coastal Zone Color Scunner data: Journal of Geophysical Research, v. 94, no. C9, p. 12681-12691.

Havelock, D.I., 1989, Geometric precision in noise-free digital images: IEEE [Institute of Electrical and Electronics Engineers] Transactions on Pattern Analysis and Machine Intelligence, v. 11, no. 10. p. $1065-1075$.

Leese, J.A., Novak, C.S., and Clarke, B.B., 1971, An automated technique for obtaining cloud motion for geosynchronous satellite data using cross correlation: Journal of Applied Meteorology, v. 10, no. 1, p. 118-132.

Li, M., 1990, High-precision relative orientation using feature-based matching techniques: ISPRS [International Society for Photogrammetry and Remote Sensing] Journal of Photogrammetry and Remote Sensing, v. 44, no. 6, p. 311-324.

Lyvers, E.P., Mitchell, O.R., Akey, M.L., and Reeves, A.P., 1989, Subpixel measurements using a moment-based edge operator: IEEE Transactions on Pattern Analysis and Machine Intelligence, v. 11, no. 12, p. 1293-1309.

McGill, S.F., Allen, C.R., Hudnut, K.W., Johnson. D.C., Miller, W.F., and Sieh, K.E., 1989. Slip on the Superstition Hills fault and on nearby faults associated with the 24 November 1987 Elmore Ranch and Superstition Hills earthquakes, southern California: Bulletin of the Seismological Society of America, v. 79 , no. 2, p. 362-375.

Morgan, J.S., Slater, D.C., Timothy, J.G.. and Jenkins, E.B., 1989. Centroid position measurements and subpixel sensitivity variations with the MAMA detector: Applied Optics, v. 28, no. 6, p. 1178-1192.

Mort, M.S., and Srinath, M.D., 1988, Maximum likelihood image registration with subpixel accuracy, in Tescher, A.G., ed. Applications of digital image processing XI: SPIE [Society of Photo-Optical Instrumentation Engineers] Proceedings, v. 974, p. 38-45. 
Newhall, C.G., Sharp, R.V., Wieczorek, G.F., Wennerberg, L., and Bicknell, J.D., 1990, The July 16, 1990, Luzon earthquake: U.S. Geological Survey and Philippine Institute of Volcanology and Seismology, unpublished final report, $59 \mathrm{p}$.

Ninnis, R.M.. Emery, W.J., and Collins, M.J., 1986, Automated extraction of pack ice motion from Advanced Very High Resolution Radiometer imagery: Journal of Geophysical Research, v, 91, no, C9, p. 10725-10734.

Qi Tian and Huhns, M.N., 1986, Algorithms for subpixel registration: Computer Vision, Graphics, and Image Processing, v. 35, no. 2, p. 220-233.

Shelton, J.S., 1966, Geology illustrated: San Francisco, W.H. Freeman and Company, $434 \mathrm{p}$.

Stern, M., 1987, A new tool: SPOT imagery for studying rapid movements. in Symposium on Remote Sensing of Environment, 21st, Ann Arbor, Michigan, USA, 1987, Proceedings: Ann Arbor, Michigan, USA, Environmental Research Institute of Michigan, v. 2, p. 917-924.

Sutton, M.A., McNeill, S.R., Jang, J., and Babai, M., 1988, Effects of subpixel image restoration on digital correlation error estimates: Optical Engineering, v. 27 , no. 10 , p. $870-877$.

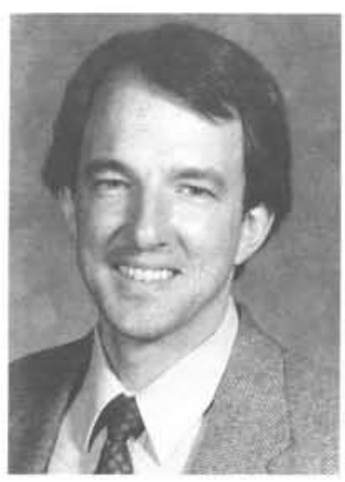

Dr. Robert E. Crippen is a Research Geologist at the National Aeronautics and Space Administration's Jet Propulsion Laboratory, California Institute of Technology, Pasadena, California 91109, USA. He has developed new approaches to the extraction of geologic information from remotely sensed imagery and other spatial data sets. His primary research centers on neotectonic activity, particularly for sites in the arid lands of the southwestern United States. 